\title{
Wideband Coffee-Bean Shaped Radiating Element for Circularly-Polarized Waveguide Slot Arrays
}

\author{
Miguel Ferrando-Rocher ${ }^{1,2}$, Jose I. Herranz-Herruzo ${ }^{1}$, Alejandro Valero-Nogueira ${ }^{1}$ \\ ${ }^{1}$ Antennas and Propagation Laboratory (APL-iTEAM), Universitat Politècnica de València, Spain \\ ${ }^{2}$ Microwave and Applied Computational Electromagnetics Group (GMECA), Universidad de Alicante, Spain
}

\begin{abstract}
A wideband coffee-bean shaped radiating element for circularly-polarized waveguide slot arrays is presented. The proposed solution is based on a very simple polarizer aimed to be integrated into flat panel antennas in the millimeter-wave band. For validation purposes, a slot array fed by a groove gap waveguide network at $30 \mathrm{GHz}$ is taken as a feeder. Simulated results using periodic boundary conditions in a $2 \times 2$ unit cell are presented in this work. Preliminary results show a frequency bandwidth of $4 \mathrm{GHz}$ with input reflection coefficient better than $-10 \mathrm{~dB}$. It is worth stressing the good polarization purity achieved, being below $1.5 \mathrm{~dB}$ from $29 \mathrm{GHz}$ to $31 \mathrm{GHz}$ and below $3 \mathrm{~dB}$ in the whole band of interest ( 28 to $32 \mathrm{GHz}$ ).
\end{abstract}

Index Terms-Circular polarization, Coffee-Bean Polarizer, Gap waveguide technology, Ka-Band, Slotted-waveguide antenna

\section{INTRODUCTION}

Circularly polarized (CP) antennas in the millimeter-wave band have recently caught the spotlight of industry because of their appealing features, such as increased usable bandwidth, high load capacity and the ability to support instantaneous transmission of massive data [1]. Moreover, 5G mm-wave systems are today a hot-topic and they are eager of low-profile, easy fabrication, directional radiation, and high gain solutions.

Facing this context and as stated in [2]: there exist three well-known strategies for forming a planar broadband CP antenna array suitable for the $\mathrm{mm}$-wave band: sequential rotation schemes [3], broadband CP radiating elements fed by full-corporate networks [4], [5] or linearly polarized (LP) radiating elements along with an LP-to-CP converter. This paper focuses on a strategy following this latter option.

LP-to-CP converter method becomes attractive for flat panel array antennas (FPA). In this way, it is easy to place the polarizer directly on the FPA and transform the linear polarization into CP. Essentially, the polarizer decomposes the LP incident plane wave into two orthogonal components of nearly equal amplitude with a phase difference of $90^{\circ}$ to generate a $\mathrm{CP}$ transmitted plane wave.

A very simple and low-profile polarizer that can be easily integrated into a FPA is here presented. Basically, the polarizer consists of a circular patch, split into two equal parts slightly shifted, which achieves a very interesting modal characteristic. The novelty of this polarizer lies in its simplicity, low cost and ease of manufacture and integration into FPAs, with possible applications for Ka-band satellite communications.
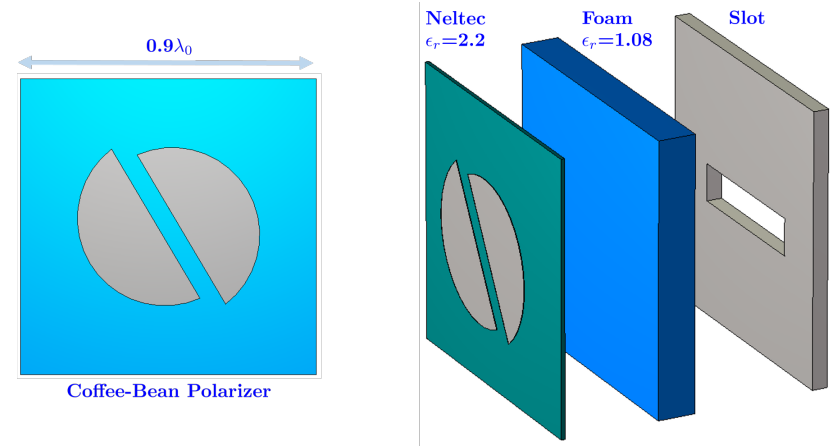

Fig. 1: Perspective view of the radiating element.

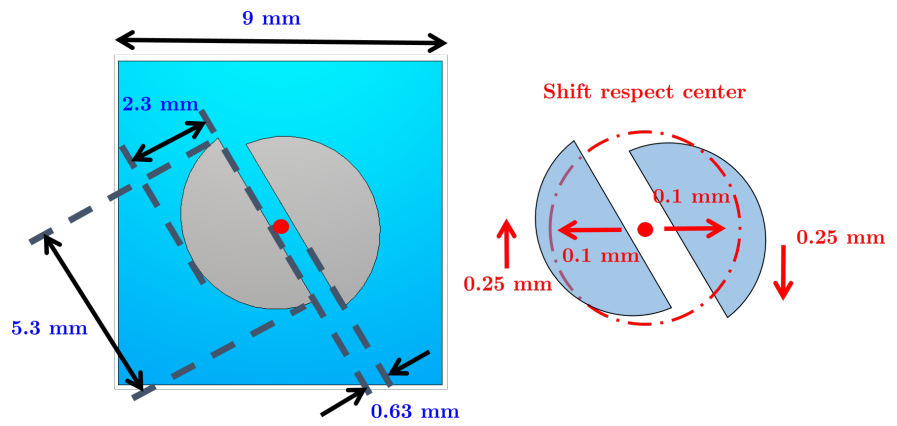

Fig. 2: Top view of the coffee-bean shaped polarizer where the relevant dimensions of the structure are indicated.

\section{UNIT CELL WORKING PRINCIPLE}

The basic cell consists of a structure with dimensions $0.9 \times 0.9 \lambda_{0}$. The free-space wavelength $\left(\lambda_{0}\right)$ corresponds to the central working frequency, in this case $10 \mathrm{~mm}$.

The layers of the polarizer, which are shown in Fig. 1, are stacked as follows. A rectangular slot acts as a feeder. On top rests a foam layer with a permittivity of 1.08 and a thickness of $1.22 \mathrm{~mm}$ and a Neltec dielectric sheet with a dielectric constant $\epsilon_{r}$ of 2.2 and $128 \mu \mathrm{m}$ thickness. On top of this latter layer, the metallic coffee-bean shape with a thickness of $20 \mu \mathrm{m}$ is printed. The resulting shape of this coffee-bean polarizer is composed of a metal circle in which a rectangular metal area is removed, leading to two disjointed metal segments facing each other. These two disjointed metal pieces allow an additional degree of freedom in the design since the centers of the two semicircles can be different. All the geometric details of the polarizer are shown in Fig. 2. 

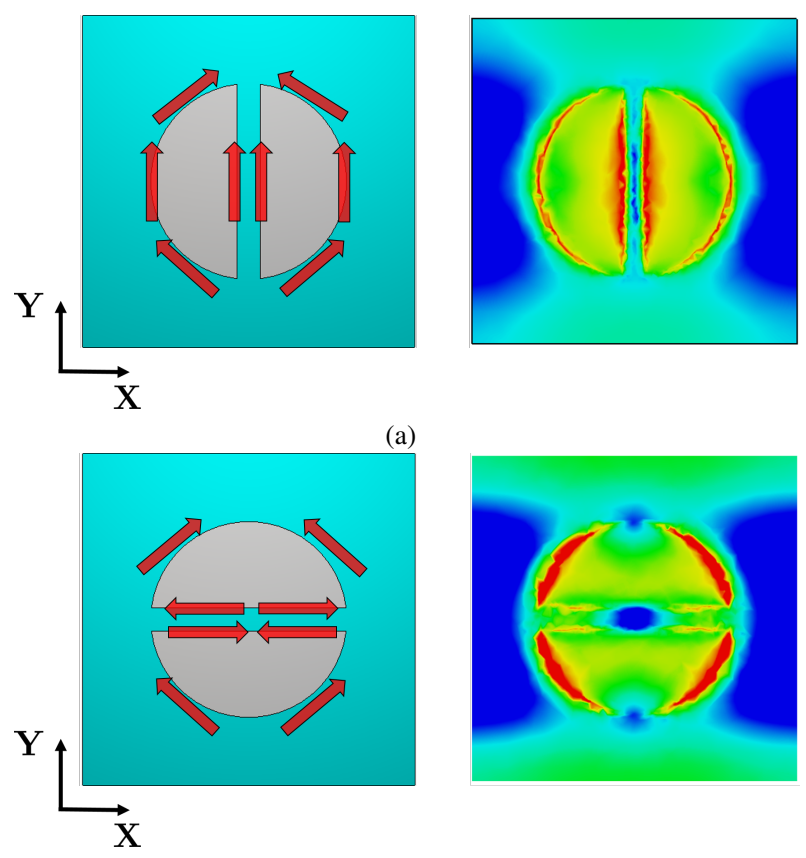

(b)
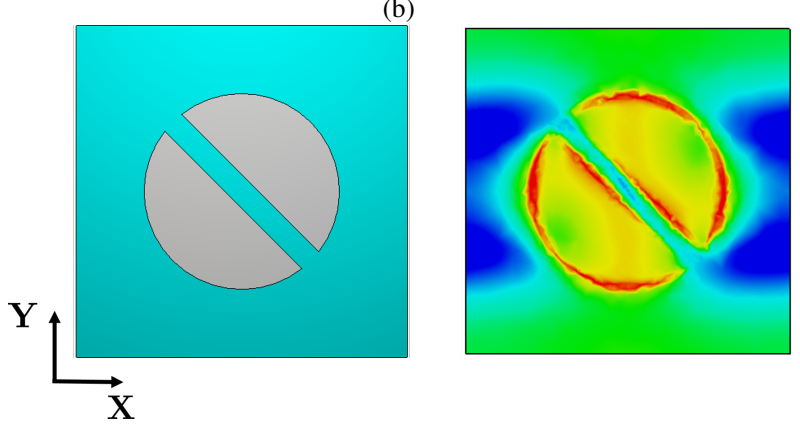

(c)

Fig. 3: Current distribution for different positions of the coffee pods.

\section{A. Study of the fields in the unit cell}

It can be shown that the described metallic shape supports two dominant modes, one for each polarization of the field.

Fig. 3a shows the current distribution when the illuminating electric field is parallel to the central slot. It can be seen that the intensity is mainly concentrated around the central gap. Fig. $3 \mathrm{~b}$ shows the current distribution when the illuminating field is perpendicular to the central slot. In this second case it can be seen that the radiating currents are concentrated along the outer contour of both metal segments. Finally, Fig. 3c shows how the central slot is rotated $45^{\circ}$ with respect to the illuminating field. This rotation achieves an overlap of the modes shown in Fig. 3a and Fig. 3b.

Note that Fig. 3a and Fig. 3b show the specific direction of the field as the polarisation of the currents remains constant for any given time instant. This is not the case in Fig. 3c where the current changes with time. Fig. 4 shows the temporal variation of the tangential component of the electric field on the surface of the polarizer. Specifically, 6 different time
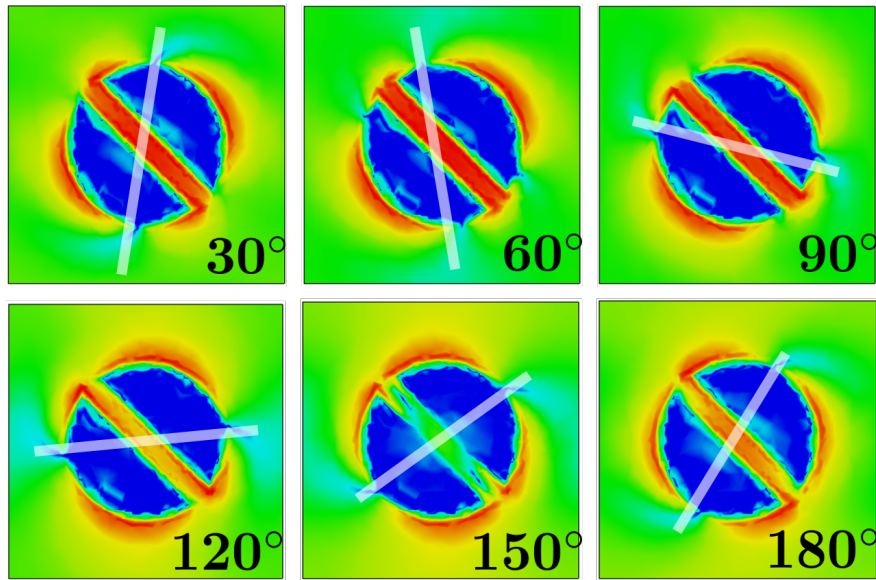

Fig. 4: Temporal variation of the E-field tangential component on the surface of the polarizer.

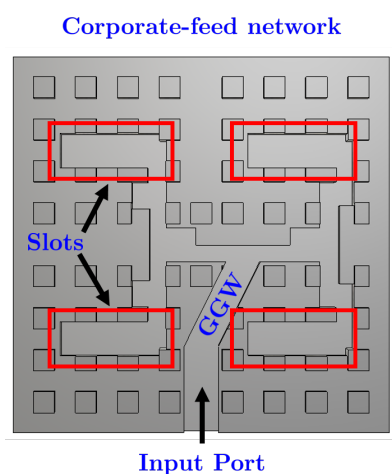

Fig. 5: $2 \times 2$ array structure.

instants are shown, from $30^{\circ}$ to $180^{\circ}$ in $30^{\circ}$ steps.

\section{ARRAY SIMULATED PERFORMANCE}

To validate the performance of the polarizer, the unit cell shown in Fig. 5 is then used as a feeder. In the left side of the figure, a top view of the corporate-feed distribution network using horizontally-polarized Groove Gap Waveguide (GGW) [6] is shown. This compact chandelier-shape distribution network could also be scaled to feed a larger array. Septums on the power-dividers to maximize signal matching are used. Moreover, the GGW crosses diagonally from the input port to the first power-divider to feed all the elements in phase and also squeezing the limited space available. On the right side of Fig. 5, an exploded view of all the layers that make up the basic cell is shown: GGW feed network, slots and polarizer.

Now, some preliminary results are shown. Fig. 6 exhibits the radiation patterns in $\mathrm{XZ}$ and YZ-planes. Radiation patterns correspond to an $8 \times 8$ array. Periodic boundary conditions around the $2 \times 2$ unit cell (Fig. 5) have been used in the simulation. A great stability of the radiation patterns and perfect agreement with a uniform illumination is observed in both cuts. Naturally, as the frequency increases grating lobes 

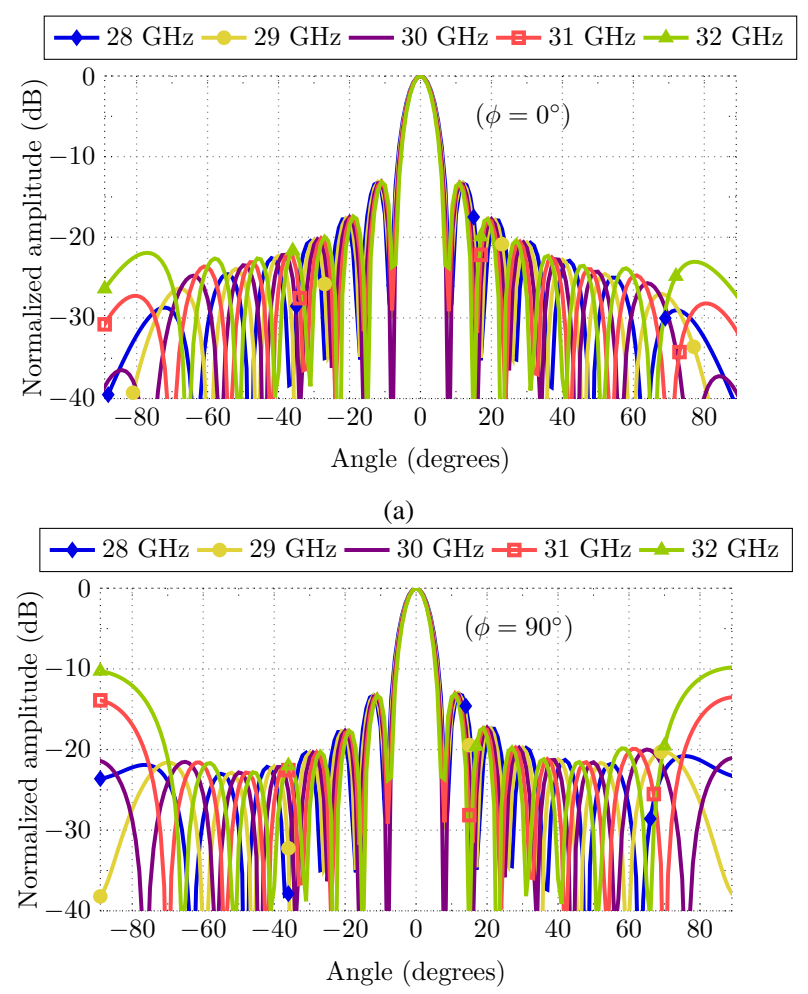

(b)

Fig. 6: Simulated RHCP radiation patterns: (a) $\phi=0^{\circ}$ plane; (b) $\phi=90^{\circ}$ plane.

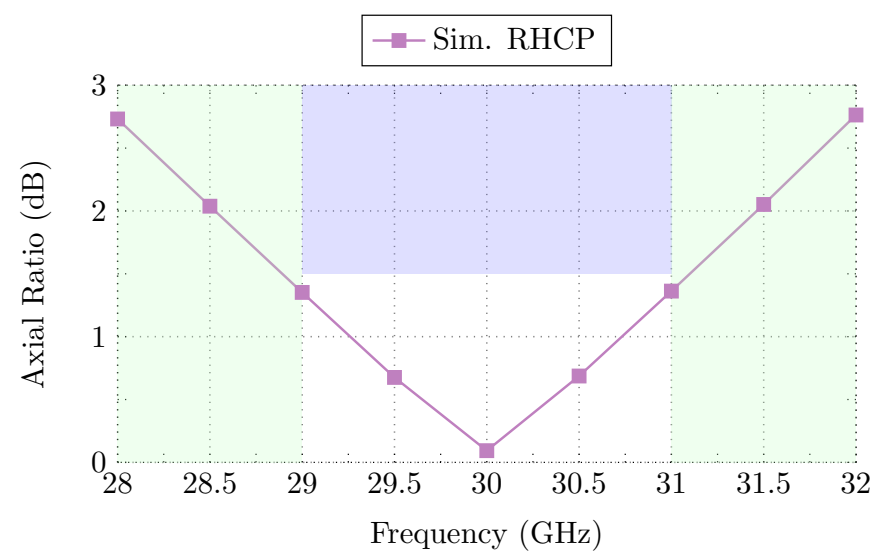

Fig. 7: Simulated axial ratio.

appear in the $\phi=90^{\circ}$ plane (YZ-plane), however this could be mitigated using strategies similar to those used in [4], [7]. In addition, it should be noted that the $4 \mathrm{GHz}$ bandwidth shown, corresponds to a $13.3 \%$ of relative bandwidth.

Fig. 7 shows the axial ratio (AR) of the simulated array. It stands out the circular polarization purity at $30 \mathrm{GHz}$. In addition, two areas are shaded in the figure. The center area limits the frequency range with an axial ratio lower than $1.5 \mathrm{~dB}$. The edge shaded areas indicate that an AR lower than $3 \mathrm{~dB}$ is obtained for the whole operating frequency range (28 to $32 \mathrm{GHz}$ ). Finally, the reflection coefficient is presented in

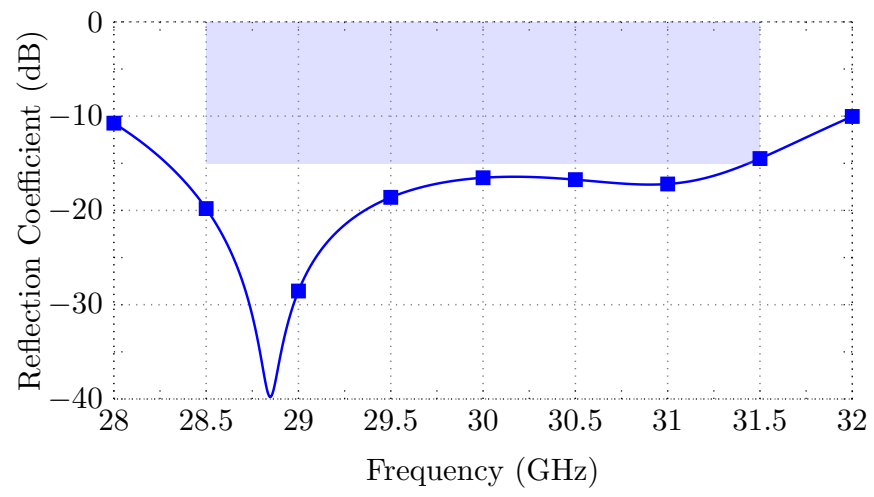

Fig. 8: Simulated reflection coefficient.

Fig. 8. In this case the area where the $\mathrm{S}_{11}$ is below $-15 \mathrm{~dB}$ is shaded from $28.5 \mathrm{GHz}$ to $31.5 \mathrm{GHz}$, being lower than -10 $\mathrm{dB}$ in the entire working band.

\section{CONCLuSion}

A coffee-bean shaped printed element, aimed to transform the linearly-polarized radiated field of a slot array into a circularly-polarized one, is designed in Ka-band. The behavior of the currents on the polarizer is explained, in order to describe its good performance with a 3-dB axial-ratio bandwidth of more than $13 \%$. In addition, a unit cell implemented in Gap Waveguide technology is used to feed the conceived polarizer. The GGW chandelier-type network employed can be extended to feed larger arrays. The polarizer can be a good solution for low-cost and easy integration into FPAs with height and weight restrictions.

\section{ACKNOWLEDGMENT}

This work has been supported by the Spanish Ministry of Science, Innovation and Universities (Ministerio de Ciencia, Innovación y Universidades) under project PID2019107688RB-C22.

\section{REFERENCES}

[1] K. X. Wang and H. Wong, "A wideband millimeter-wave circularly polarized antenna with 3-d printed polarizer," IEEE Transactions on Antennas and Propagation, vol. 65, no. 3, pp. 1038-1046, 2017.

[2] Q. Wu, J. Hirokawa, J. Yin, C. Yu, H. Wang, and W. Hong, "Millimeterwave planar broadband circularly polarized antenna array using stacked curl elements," IEEE Transactions on Antennas and Propagation, vol. 65 , no. 12, pp. 7052-7062, 2017.

[3] Q. Zhu, K.-B. Ng, and C. H. Chan, "Printed circularly polarized spiral antenna array for millimeter-wave applications," IEEE Transactions on Antennas and Propagation, vol. 65, no. 2, pp. 636-643, 2016.

[4] M. Ferrando-Rocher, J. I. Herranz-Herruzo, A. Valero-Nogueira, and A. Vila-Jiménez, "Single-layer circularly-polarized $k a$-band antenna using gap waveguide technology," IEEE Transactions on Antennas and Propagation, vol. 66, no. 8, pp. 3837-3845, 2018.

[5] M. Ferrando-Rocher, A. Valero-Nogueira, J. I. Herranz-Herruzo, and J. Teniente, "60 ghz single-layer slot-array antenna fed by groove gap waveguide," IEEE Antennas and Wireless Propagation Letters, vol. 18, no. 5, pp. 846-850, 2019. 
[6] M. Ferrando-Rocher, A. Valero-Nogueira, J. I. Herranz-Herruzo, A. Berenguer, and B. Bernardo-Clemente, "Groove gap waveguides: A contactless solution for multilayer slotted-waveguide array antenna assembly," in 2016 10th European Conference on Antennas and Propagation (EuCAP). IEEE, 2016, pp. 1-4.

[7] M. Ferrando-Rocher, J. I. Herranz-Herruzo, A. Valero-Nogueira, and M. Baquero-Escudero, "Flatness enhancement of gap waveguide slot arrays using a ribbed-grid plate," in 2018 IEEE International Symposium on Antennas and Propagation \& USNC/URSI National Radio Science Meeting. IEEE, 2018, pp. 419-420.

This paper's copyright is held by the author(s). It is published in these proceedings and included in any archive such as IEEE Xplore under the license granted by the "Agreement Granting EurAAP Rights Related to Publication of Scholarly Work." 